\title{
Prevalência de enteroparasitos em moradores da cidade de Cáceres/MT
}

Dentre os diversos problemas que assolam a saúde pública no Brasil, o enteroparasitismo se mostra como um dos mais sérios, apresentando forte correlação com aspectos de desnutrição das populações, podendo afetar o desenvolvimento físico, psicossomático e social das pessoas. A transmissão desses parasitas está relacionada com as condições de vida e higiene das comunidades, ocorrendo alta prevalência em populações de baixo nível socioeconômico, geralmente desprovidas de infraestrutura sanitária mínima. Os estudos sobre parasitos intestinais no Brasil são fragmentados e para o estado de Mato Grosso, especialmente o município de Cáceres, são raros. Assim este estudo objetivou diagnosticar a prevalência de parasitos intestinais na população acima de cinco anos de idade, em dois bairros periféricos no município de Cáceres/MT, um dos 100 municípios mais pobres do país. Entre janeiro e abril de 2013, coletores estéreis descartáveis foram distribuídos nas residências e recolhidos no dia seguinte e as amostras submetidas aos exames coprológicos, empregando a técnica de sedimentação espontânea. Os resultados foram analisados quanto às prevalências dos enteroparasitos em crianças e adultos. Do total das amostras analisadas, $74,86 \%$ foram positivas para algum tipo de parasito. O número médio de espécies presentes por amostra foi de 1,69 parasitos sendo registrado poliparasitismo em $26,78 \%$ das amostras. $O$ índice de prevalência de enteroparasitos foram considerados altos, diante das médias nacionais e internacionais, havendo predominância de ocorrência de protozoários, revelando a necessidade de maiores investimentos públicos locais.

Palavras-chave: Doenças Parasitárias; Helmintos; Protozoários.

\section{Prevalence of enteroparasites in residents of the city of Cáceres/MT}

Among the many problems that afflict public health in Brazil, enteroparasitism is one of the most serious, presenting a strong correlation with the aspects of malnutrition of the populations, which can affect the physical, psychosomatic and social development of the people. The transmission of these parasites is related to the living conditions and hygiene of the communities, occurring high prevalence in populations of low socioeconomic level, generally lacking a minimum sanitary infrastructure. Studies on intestinal parasites in Brazil are fragmented and for the state of Mato Grosso, especially the municipality of Cáceres, are rare. Thus, this study aimed to diagnose the prevalence of intestinal parasites in the population above five years of age, in two peripheral neighborhoods in the city of Cáceres/MT, one of the 100 poorest municipalities in the country. Between January and April 2013, sterile disposable collectors were distributed in the residences and collected the following day the samples and submitted to the coprological exams, using the technique of spontaneous sedimentation. The results were analyzed regarding the prevalence of enteroparasites in children and adults. Of the total samples analyzed, $74.86 \%$ were positive for some type of parasite. The mean number of species present per sample was 1.69 parasites, and polyparasitism was recorded in $26.78 \%$ of the samples. The prevalence rate of enteroparasites was considered high in relation to national and international averages, with a predominance of protozoa occurrence, indicating the need for greater local public investments.

Keywords: Parasitic Diseases; Helminths; Protozoans.

Topic: Epidemiologia e Saúde Ambiental

Reviewed anonymously in the process of blind peer.
Received: $10 / 04 / 2018$

Approved: 24/05/2018
Joselaine Souto Hall Silva (iD)

Universidade do Estado de Mato Grosso, Brasil http://lattes.cnpq.br/2607621597856798 http://orcid.org/0000-0001-5667-8871 joselaineshs@unemat.br

Dionei José da Silva (iD)

Universidade do Estado de Mato Grosso, Brasil http://lattes.cnpq.br/5447213851327253

http://orcid.org/0000-0002-6189-9756

dioneijs@unemat.br

Jeffrey Jon Shaw (iD

Universidade de São Paulo, Brasil http://lattes.cnpq.br/4665897914902853

http://orcid.org/0000-0002-7008-1972

jayyusp@hotmail.com

\author{
Antonio Francisco Malheiros (iD) \\ Universidade do Estado de Mato Grosso, Brasil \\ http://lattes.cnpq.br/9067970026570376 \\ http://orcid.org/0000-0001-8169-0557 \\ malheiros@unemat.br
}

\section{Referencing this:}

SILVA, J. S. H.; SILVA, D. J.; SHAW, J. J.; MALHEIROS, A. F.. Prevalência de enteroparasitos em moradores da cidade de Cáceres/MT. Revista Ibero Americana de Ciências Ambientais, v.9, n.4, p.154-164, 2018. DOI: http://doi.org/10.6008/CBPC2179-6858.2018.004.0013 


\section{INTRODUÇÃO}

Dentre os diversos problemas que assolam a saúde pública nos países pouco desenvolvidos, o parasitismo intestinal se revela como um dos mais sérios. Apresenta forte correlação com desnutrição das populações, podendo afetar o desenvolvimento físico, psicossomático e social das pessoas, principalmente daquelas em fase escolar (LANDER et al., 2012). Estima-se que atualmente exista, no mundo, entre um a 3,5 bilhões de pessoas contaminadas por algum tipo de enteroparasito (PRESTES et al., 2015). Destas, aproximadamente 450 milhões apresentam quadros sintomáticos, sendo a maioria crianças (PRESTES et al., 2015). Na América Latina estima-se que $30 \%$ da população esteja contaminada por algum tipo de enteroparasito e para o Brasil a frequência pode chegar a 80\% em algumas regiões (FONSECA et al., 2010).

A contaminação por parasitos está relacionada com as condições de vida e higiene das comunidades, ocorrendo alta prevalência na população de baixo nível socioeconômico, principalmente entre as crianças (DUNCAN et al., 2013). O agravamento de infecções pode se dar pelo crescimento acelerado dos centros urbanos, levando ao estabelecimento de comunidades marginais e grandes aglomerações humanas, geralmente desprovidas de infraestrutura sanitária mínima, onde se criam condições ótimas para transmissão dos enteroparasitos (DUNCAN et al., 2013).

Grande parte dos casos de enteroparasitoses não são diagnosticados, visto que muitas vezes podem ser assintomáticos, o que dificulta a determinação de sua prevalência e o controle de sua transmissão. A fim de buscar diagnósticos preventivos e em decorrência dos efeitos deletérios à saúde dos indivíduos e, sobretudo, das repercussões econômicas, vários programas foram dirigidos para o controle das parasitoses intestinais em diferentes países, mas no Brasil a maior parte dos estudos ainda são pontuais (VISSER et al., 2011).

Para o Estado de Mato Grosso e especialmente o município de Cáceres, são raros os levantamentos coproparasitológicos. Assim este estudo contribui sobremaneira para o conhecimento da realidade acerca da epidemiologia das enteroparasitoses da população local, apresentando uma importante contribuição para tomada de decisões e ações direcionadas à saúde pública. Enquanto diagnóstico, constitui subsídio importante para tomada de decisões e implantação de políticas públicas, principalmente quando se verifica que Cáceres/MT figura entre os 100 municípios mais pobres do país (LACERDA, 2017) e como consequência em muitos bairros não há estruturas mínimas de saneamento básico. Assim, buscamos conhecer a prevalência de enteroparasitoses, através de exames coproparasitológicos, na população acima de cinco anos de idade da cidade de Cáceres/MT, tomando dois bairros como referência.

\section{MATERIAIS E MÉTODOS}

O município de Cáceres, localizado na região Oeste do estado de Mato Grosso, apresenta população de 90.158 habitantes (LACERDA, 2017). Os bairros selecionados para o estudo, Junco e Marajoara, estão em regiões periféricas, carentes de infraestrutura sanitária e habitado por população com baixo padrão econômico e educacional. 
As amostragens foram realizadas durante os meses de janeiro a abril de 2013. Coletores descartáveis de plástico, estéreis, foram entregues nas residências e orientações de coleta e armazenamento do material fecal foram repassadas a um morador adulto. Por três dias consecutivos as residências foram revisitadas para recolhimento das amostras, que foram submetidas aos exames coproparasitológicos, empregando a técnica de sedimentação espontânea (HOFFMAN, 1934).

Foram calculadas as frequências das enteroparasitoses em geral, dos diferentes enteroparasitos e do poliparasitismo intestinal. A distribuição dessas prevalências foi analisada quanto aos grupos de crianças e adultos. Testada a normalidade dos dados, foi realizado o teste $t$ de Student para dois grupos a fim de verificar a ocorrência de diferenças estatisticamente significativas entre o número de registros de enteroparasitoses para: protozoários $\mathrm{x}$ helmintos; total de crianças positivas $\mathrm{x}$ total de adultos positivos. Foi considerando estatisticamente significativo quando $p$ foi menor que 0,05 . Este estudo foi submetido à avaliação de Comitê de Ética em Pesquisa com base nas leis nacionais: CEP-ICB/USP-310.501/2013.

\section{RESULTADOS}

Ao todo foram obtidas 183 amostras, sendo 95 de adultos e 88 de crianças entre cinco e 15 anos. Submetidas aos exames coprológicos, registramos 11 espécies de enteroparasitos, dos quais seis eram protozoários (Entamoeba coli, Entamoeba histolytica, Endolimax nana, Chilomastix mesnili, Giárdia lamblia e Blastocystis spp.) e cinco helmintos (Ascaris lumbricoides, Enterobius vermiculares, Hymenolepis nana, Strongyloides stercoralis e Trichuris trichiura). $74,86 \%(n=137)$ das amostras foram positivas para algum tipo de parasito. A ocorrência de protozoários foi significativamente maior que de helmintos $(t=4,298$; $d f=5$; $p=0,008)$, sendo que $72,68 \%(n=133)$ das amostras foram positivas para alguma espécie de protozoário; 23,50\% ( $n=43)$ para alguma espécie de helminto e $21,31 \% \quad(n=39)$ para protozoário e helminto concomitantemente.

O número médio de espécies presentes por amostra foi de 1,69 parasito por pessoa, sendo que para crianças foi de 1,95 enquanto para adultos foi de 1,43. No geral, o poliparasitismo prevaleceu, estando presente em $26,78 \%$ das amostras. No entanto, foi mais frequente em crianças $(35,23 \%)$ e em adultos prevaleceu o monoparasitismo (26,32\%) (Figura 1). Os protozoários foram, em geral, mais frequentes que helmintos, ocorrendo em mais de $10 \%$ das amostras, exceto pelo protozoário Blastocystis spp. que ocorreu em 4,92\% ( $n=9)$ e pelo helminto $H$. nana que ocorreu em $14,21 \%(n=26)$ das amostras (Figura 2 ).

O índice de positividade para crianças e adultos, não variou significativamente entre as duas classes etárias ( $t=1,1930 ; d f=11 ; p=0,080)$, embora tenha-se encontrado maior ocorrência em geral, para crianças $(79,55 \%)$ do que para adultos (70,53\%). Os enteroparasitos E. nana, C. mesnili e $E$. coli, foram os mais frequentes, ocorrendo em mais de $27 \%$ das amostras, tanto em adultos como em crianças. G. lamblia, $\mathrm{H}$. nana e E. hystolitica apresentaram frequência acima de $10 \%$ em crianças e os demais parasitos apresentaram frequência menor que $10 \%$ nas duas faixas etárias. Exceto para Blastocystis spp. em que houve 7,37\% ( $n=7$ ) de positividade para adultos e $2,27 \%(n=2)$ para crianças, em todos demais casos os parasitos foram mais frequentes em crianças (Figura 3 ). 


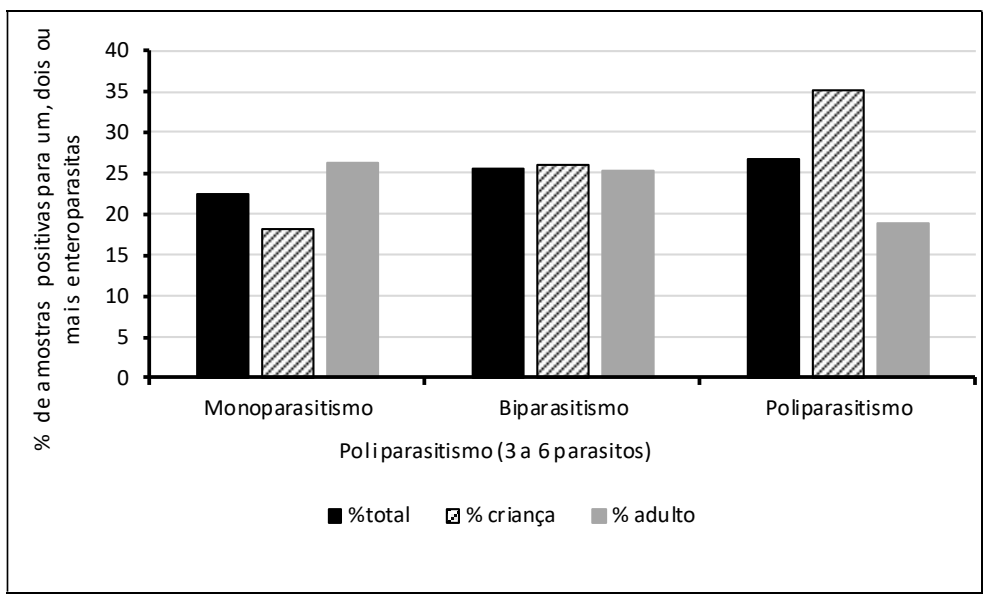

Figura 1: Percentual de casos de mono, bi e poliparasitismo em amostras da população de crianças e adultos oriundos do município de Cáceres/MT.

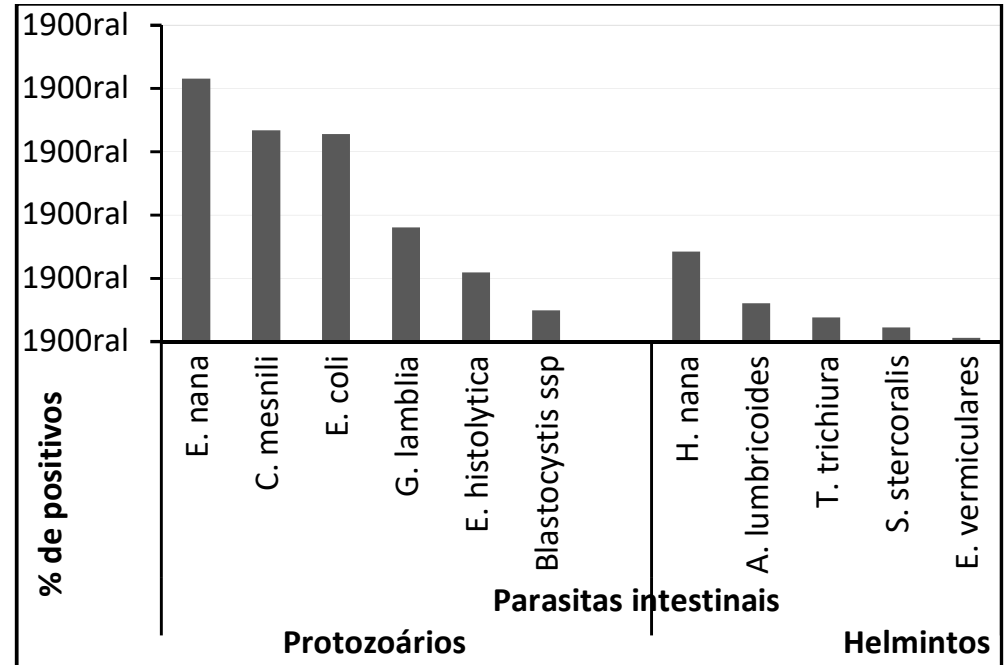

Figura 2: Percentual de positivos para Protozoários e Helmintos do total de 183 amostras - Cáceres/MT.

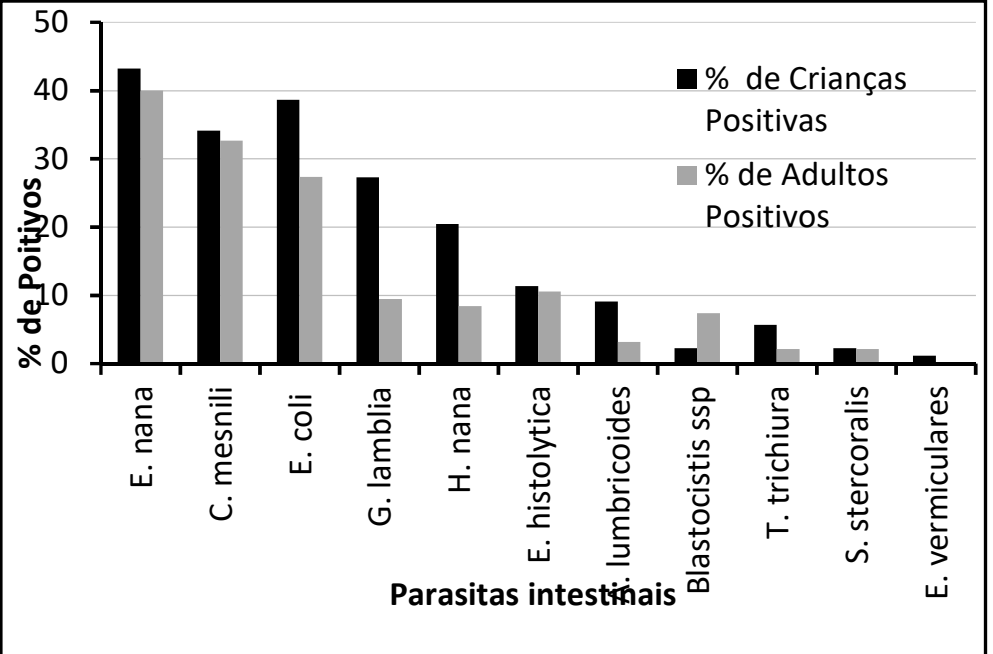

Figura 3: Percentual de positividade de enteroparasitos em crianças e adultos em Cáceres/MT.

\section{DISCUSSÃO}

Estudos sobre prevalência de enteroparasitoses, realizados em diferentes países, revelaram frequências variando de 14,6\% a 90\% (MACHADO et al., 2008). As parasitoses intestinais são mais frequentes em regiões menos desenvolvidas, aumentando na medida em que piora as condições socioeconômicas, atingindo, em algumas localidades, índices de até 90\% da população (LUDWIG et al., 1999). Esta relação foi 
verificada em diversas regiões brasileiras, mostrando maiores incidências onde há precárias condições de saneamento, com altas taxas de infecção de até 88,4\% (MACHADO et al., 2008).

A frequência de enteroparasitoses que encontramos (74,86\%) está próxima aos índices máximos registrados para o Brasil (MACHADO et al., 2008). Índices bem inferiores foram observados, por exemplo em estudos que avaliaram a correlação entre condições de saneamento básico e parasitoses intestinais na população de Assis-SP, em que foi registrado 23,3\% de incidência de enteroparasitoses (LUDWIG et al., 1999). Taxas também inferiores $(51,4 \%)$ foram encontradas na população de Tangará da Serra/MT (TIAGO et al., 2005). Em Abadia de Dourados-MG foi registado índice de 47,1\% (MACHADO et al., 2008) e num estudo abordando geohelmintíase abrangendo 22 municípios brasileiros o índice médio registrado foi de 36,5\% (FONSECA et al., 2010).

A literatura é unânime em afirmar que as enteroparasitoses são mais frequentes em crianças (LUDWIG et al., 1999), o que foi observado neste estudo. No entanto, os índices que registramos em crianças em Cáceres/MT são significativamente mais elevados do que os encontrados em outros estudos realizados em Mato Grosso (TIAGO et al., 2005), onde foi registrado índice de 53\% e outros Estados Brasileiros (MONTEIRO et al., 1988; FERREIRA et al., 2005; LANDER et al., 2012) em que a taxa de registro de enteroparasitos em crianças variou de 11,5 a 30,09\%. As maiores incidências em crianças são favorecidas por estas apresentarem menores cuidados com os alimentos, a higiene e maior contato com solo, animais domésticos, brinquedos e outros objetos que uma vez contaminados acaba por contaminar as crianças (LUDWIG et al., 1999; SANTOS et al., 2010). Outro fator que explica a menor incidência em adultos que em crianças é a possível imunidade progressiva e duradoura desenvolvida com o aumento da idade, particularmente para o A. lumbricoides (LUDWIG et al., 1999).

Embora a frequência de enteroparasitos tenha sido numericamente maior em crianças, não há diferença estatisticamente significativa entre índices de frequência em crianças e adultos. Esta proximidade e frequências altas, em todas faixas etárias, indicam infecção em nível familiar e evidência que os cuidados de higiene dos adultos estão aquém do ideal, reflexo do baixo nível sociocultural e do déficit em infraestrutura básica de saneamento local.

A inexistência de pavimentação, rede de esgoto e falta de rede de água tratada em muitas residências, constituem fatores determinantes para a alta prevalência de parasitoses na população analisada em Cáceres/MT. Condição similar foi encontrada em municípios do Estado de São Paulo (MONTEIRO et al., 1988; MACHADO et al., 2008) em estudos que relacionaram condições de saneamento básico e parasitoses intestinais na população dos municípios de São Paulo e Assis. Nestes locais foi observada considerável redução do índice de enteroparasitoses logo após a população ter sido beneficiada por sistema de água tratada e esgoto (MONTEIRO et al., 1988; MACHADO et al., 2008).

O número médio de espécies de enteroparasitos presentes por amostra, na população de CáceresMT foi elevado, em comparação com outros estudos. Enquanto registramos 1,69 parasitos por pessoa, com média de 1,95 para crianças e 1,43, para adultos, a maioria dos estudos apontam média de 1,1 parasito por pessoa (FERREIRA et al., 2005). Casos de bi e poliparasitismo são frequentes (MACHADO et al., 2008), os quais 
agravam o estado de saúde da pessoa parasitada e tendem a aumentar na medida em que diminui o nível socioeconômico da população (MONTEIRO et al., 1988). Neste estudo os índices de bi e poliparasitismo foram considerados altos, aparecendo respectivamente em $25,68 \%$ e $26,78 \%$ das amostras, enquanto estudos conduzidos em municípios de Minas Gerais (MACHADO et al., 2008) e São Paulo (MONTEIRO et al., 1988) este índice foi de 8,8\% e no Paraná (FERREIRA et al., 2005) de 3,2\% das amostras.

Quando considerado apenas as crianças, a frequência de mais de um parasito por pessoa foi ainda mais acentuada, aparecendo biparasitismo em $26,14 \%$ e poliparasitismo em $35,23 \%$ das amostras. $O$ alto índice de poliparasitismo que encontramos indica que a população amostrada se encontra exposta a diversas condições e fatores que favorecem a contaminação por enteroparasitos, a maioria deles relacionados a fatores culturais, econômicos e carência de infraestrutura pública. Desta forma, é evidente a necessidade de implementação de políticas públicas, não apenas em medidas curativas, mas preventivas, no sentido de proporcionar melhoria na qualidade socioeconômica da população.

O reiterado registro dos enteroparasitos E. histolytica, G. lamblia, A. lumbricoides e $T$. trichiura, em nosso estudo, era esperado tendo em vista que estes estão entre os parasitos intestinais mais frequentemente relatados na literatura (LANDER et al., 2012). No entanto, neste estudo os mais frequentes foram os protozoários $E$. nana e C. mesnili e os helmintos H. nana e A. lumbricoides.

O protozoário E. coli, embora não seja considerado patogênico, sua detecção na população é importante como um indicador, na medida em que a forma de infecção deste é semelhante à de outros parasitos patogênicos (MACHADO et al., 2008). Assim seus registros servem de indicativo dos riscos aos quais a população está submetida. Este parasito é reportado com frequência em diversos estudos, no entanto o índice de 30,79\%, aqui registrados, é superior ao da maioria dos estudos realizados no Brasil, cujas taxas variam entre 3,5 a 12,8\% (LUDWIG et al., 1999; FERREIRA et al., 2005; TIAGO et al., 2005; SANTOS et al., 2010). Por outro lado, embora a prevalência de E. histolytica tenha sido menor (10,93\%) este parasito é uma das espécies mais frequentes, aparecendo em cerca de $30 \%$ da população (RIVERO et al., 2009). E. histolytica é a principal causadora da amebíase, provocando doença invasiva, estando associada à diarreia em crianças (LANDER et al., 2012), causa de desidratação e até morbidade em crianças desnutridas.

O parasito E. nana é por vezes descrito apenas como uma ameba comensal e em frequência relativamente baixa (0,8 a17,4\%) (FERREIRA et al., 2005; TIAGO et al., 2005; CARVALHO-COSTA et al., 2007; SANTOS et al., 2010). Desta forma a alta frequência (41,53\%) aqui registrada poderia não representar preocupações para com a saúde humana. No entanto, há estudos que apontam que este enteroparasito pode causar diarreia e irritação das fendas da mucosa intestinal, evoluindo para fibrose e provocando problemas de absorção dos alimentos e consequentes deficiências nutricionais (KULIK et al., 2008). Assim a alta frequência de $E$. nana, neste estudo, sobretudo nas crianças (43,18\%), é preocupante, visto que está população já sofre com deficiências nutricionais em função do baixo poder econômico, o que poderia se agravar com as infecções.

O enteroparasito $C$. mesnili é pouco abordado na literatura, com taxa de infecção mundial em torno de 6\% (CARVALHO-COSTA et al., 2007). No entanto, em nosso estudo foi o segundo parasito mais frequente, 
encontrado em 33,33\% das amostras. Embora alguns estudiosos não o considerem patogênico, quando a infecção apresenta grande concentração, pode causar distúrbios intestinais e diarreia (CARVALHO-COSTA et al., 2007) e, portanto, no caso específico da população estuda, deve-se proporcionar cuidados, mecanismos de prevenção e combate, principalmente em crianças que estão mais vulneráveis à contaminação e aos efeitos do parasito.

O protozoário enteroparasito humano mais comum em todo o mundo é G. lamblia, ocorrendo com maior frequência em populações de baixo nível socioeconômico (PEREIRA et al., 2007). No entanto, em nosso estudo, G. Iamblia foi apenas o quarto no ranking de prevalência, com 18,3\%. Sua frequência ficou próxima aos percentuais médios registrados em países em desenvolvimento (20\%), mas bem superior às médias de países desenvolvidos, que é de 2 a 5\% (PEREIRA et al., 2007). Altas taxas de contaminação, como as aqui registradas, favorecem a disseminação do parasito, visto que G. lamblia pode ser transmitida diretamente de pessoa a pessoa dispensando a passagem pelo solo, ou através de cães e gatos que eliminam as cepas no solo (MONTEIRO et al., 1988) e assim amplia a capacidade contágio, principalmente em crianças. Esta condição justifica termos observado taxa de registro em crianças $(27,27 \%)$, três vezes maior do que em adultos. Isto é preocupante, uma vez que este parasito afeta o estado nutricional das crianças, contribuindo para condição de nanismo (CARVALHO-COSTA et al., 2007; LANDER et al., 2012).

A frequência de Blastocystis, que registramos (4,2\%) encontra-se dentro dos patamares observados para o gênero em países desenvolvidos, que é de 1,5 a 15\% (KULIK et al., 2008). No entanto, é bem inferior à incidência encontrada em populações de países em desenvolvimento, onde a prevalência pode chegar a 50\% (KULIK et al., 2008). Protozoários deste gênero apresentam ampla distribuição mundial, ocorrendo com maior frequência em regiões tropicais e subtropicais, como a região de Cáceres/MT. É um agente de patogenicidade controversa que causa infecções sintomáticas como gastroenterite, dor abdominal, náusea, vômito, meteorismo e prurido anal ou até mesmo ser assintomático, cuja infecção pode ocorrer a partir da água, animais de estimação, e vegetais crus (ROSALES et al., 2017).

As helmintoses intestinais apresentam ampla distribuição geográfica, estando entre as infecções humanas mais comuns no mundo, em especial A. lumbricoides e A. duodenalis (FERREIRA et al., 2000). A contaminação por helmintos está diretamente relacionada com o nível socioeconômico da população, considerada um problema de saúde pública em muitos países, aparecendo em elevados índices de prevalência e em alguns casos apresentam morbidade significativa (LARRÉ et al., 2014). Dentre os principais danos que podem causar estão: obstrução intestinal (A. lumbricoides), desnutrição (A. lumbricoides e $T$. trichiura) e anemia por deficiência de ferro (ancilostomídeos) sendo que as manifestações clínicas são proporcionais à carga parasitária albergada pelo indivíduo (FERREIRA et al., 2000).

Desde o início do século 20 vários estudos abordando helmintoses foram realizados no Brasil e mostram que o índice médio de prevalência reduz na medida em que há melhorias nas condições socioeconômicas (FERREIRA et al., 2000). No entanto, a taxa de infecção por helmintos que registramos em Cáceres/MT (23,5\%), embora inferior a vários outros estudos realizados no Brasil (FERREIRA et al., 2000), mostra que parcela importante da população se encontra infectada e portando merece atenção no 
tratamento direto bem como em relação à melhoria das condições sanitárias, econômicas e educacionais, como medidas preventivas.

Dentre os helmintos $A$. lumbricoides é considerado o parasito intestinal mais comum, apresentando frequência de até 39\% em algumas regiões do Brasil (FONSECA et al., 2010). Assim como em outros estudos recentes no Brasil (FERREIRA et al., 2005; SANTOS et al., 2010), a frequência de A. lumbricoides, registrada em Cáceres-MT, de 6,01\% no âmbito geral e 9,09\% em crianças foi considerada baixa diante daquelas registradas por diversos estudos realizados no país, em que foram registrados índices entre 16,4 e 53,6\% (MONTEIRO et al., 1988; CARVALHO-COSTA et al., 2007; SILVA et al., 2011). As frequências abaixo das médias, nacionalmente registradas, poderiam em princípio retratar melhorias nas condições sociais, econômicas e educacionais da população bem como maior atenção à saúde básica e aspectos de saneamento básico por parte do poder público. Porém, esta não é a realidade local visto que outros enteroparasitos registrados neste estudo, cuja infecção está diretamente relacionada ao fracasso destes aspectos, apareceram em alta frequência.

Embora a frequência de $A$. lumbricoides tenha sido baixa neste estudo, não se pode negligenciar atenção à população estudada uma vez que este helminto ocorre em pequeno número de adultos albergados, e nas pessoas infectadas geralmente não há manifestação sintomática (SILVA et al., 2011). Porém, quando em infecções maciças podem resultar em bloqueio mecânico do intestino delgado, principalmente em crianças. Este é um dos fatores que fez com que esta helmintose constituísse foco de atenção da Organização Mundial de Saúde, que em 2008 estimou que mais de 980 milhões de pessoas no mundo estariam parasitadas por esse agente (SILVA et al., 2011).

O helminto $E$. vermiculares é um parasito do trato intestinal, mas há casos de parasitismo ectópico (SANTOS et al., 2002). A frequência de registros deste parasito, neste estudo, encontra-se dentro de padrões observados para outras localidades do Brasil como São Paulo (FERREIRA et al., 2005) e Pará (SANTOS et al., 2010), onde também foram abaixo de 1\%. Esta baixa frequência e registro exclusivo em crianças, reforça dados da literatura que apontam este parasito como mais comum em crianças e prevalência relacionada ao baixo nível socioeconômico, como na área estudada, com agravamento por falta de higiene pessoal e ambiental.

Dentre os helmintos que registramos, $H$. nana foi o mais frequente $(14,21 \%)$. Este cestodeo, globalmente distribuído, apresenta maiores índices de infecções em crianças e as manifestações clínicas são por vezes assintomáticas ou ocasionando dores de cabeça, fraqueza, anorexia, dor abdominal e diarreia (LIANG et al., 2013). Assim como descrito na literatura, a frequência que registramos em crianças foi maior que em adultos (20,45 e 8,42\%). Estes valores foram considerados elevados visto que em outros estudos realizados no Brasil foram observadas frequências médias de 4,68\% (MONTEIRO et al., 1988; FERREIRA et al., 2005). Por ser um parasito capaz de completar seu ciclo sem a necessidade de um hospedeiro intermediário, a contaminação entre pessoas é facilitada, principalmente por alimento contaminado, portanto relacionada à falta de cuidados básicos de higiene. Assim o alto índice registrado em Cáceres-MT, demonstra a falta de cuidados sanitários básicos das pessoas como hábito de lavar as mãos e atenção no preparo de alimentos. 
Alguns estudos realizados no Brasil registraram baixa frequência $(0,7$ a 4,32\%) de S. stercoralis (MONTEIRO et al., 1988; SANTOS et al., 2010). Em Cáceres-MT o índice de registros esteve dentro destes patamares (2,19\%) e assim como naqueles estudos é considerado abaixo da média, visto que este parasito é bastante comum em regiões tropicas e de ampla distribuição (MONTEIRO et al., 1988). Há estudos que consideram a strongiloidíase como uma das doenças parasitárias mais comuns no Brasil, apresentando prevalência de até $82 \%$ (SUDRÉ et al., 2006). Esta controvérsia entre a consideração de um dos parasitos mais comuns no Brasil e a baixa taxa de registros em estudos como o nosso, pode estar relacionado ao método de diagnóstico.

Dentre os métodos testados para o diagnóstico de $S$. stercoralis, o sorológico tem sido o mais adequado, pois em muitos exames coprológicos ocorrem falso negativo (SUDRÉ et al., 2006), o que pode ser o nosso caso e daqueles estudos que também registraram baixa frequência para este parasito. No entanto, o registro local, mesmo que em taxas subestimadas, auxilia no mapeamento desta enteroparasitose e indica a necessidade de implementar medidas de diagnóstico mais precisas de intervenções públicas para a redução da infecção. O reconhecimento de sua circulação na população é importante, visto que embora a maioria das infecções por S. stercoralis não apresentem caráter sintomático, quando albergadas em grande concentração pode levar à morte, principalmente em pacientes imunodeprimidos (SUDRÉ et al., 2006).

Alguns estudos (FERREIRA et al., 2005; FONSECA et al., 2010) assim como o nosso, registraram baixa frequência de $T$. trichiura, com índices abaixo de 4\%. No entanto, há estimativas de que mais de um bilhão de pessoas, no mundo, estejam contaminados por este helminto (SMITH et al., 2001). Pesquisas realizadas em países das Américas Central e do Sul indicam prevalência próxima a 30\% (SMITH et al., 2001). A infecção por T. trichiura pode levar a casos de disenteria e crescimento lento em crianças, e ainda afetar o desenvolvimento de funções cognitivas (SMITH et al., 2001). Assim, mesmo em baixa frequência o seu registro indica ocorrência local e atenção deve ser dada da mesma forma que para outros enteroparasitos, pois a circulação do mesmo pode ampliar visto às características de baixa estrutura sanitária local, e complicações à saúde dos infectados, já que, crianças desnutridas podem apresentar sérias complicações quando infectadas.

\section{CONCLUSÕES}

Os altos índices de enteroparasitoses são relacionados diretamente como consequência de baixas condições sanitárias e socioculturais das populações afetadas. Estes fatores certamente influenciaram as altas taxas de registro de enteroparasitos para população de Cáceres/MT, maiores que as médias nacionais, onde grande parte do município não é atendido por rede de esgoto, água tratada e a população apresenta baixo nível socioeconômico.

As frequências elevadas de bi e poliparasitismo, superando índices nacionais e internacionais, assim como a proximidade das frequências entre crianças e adultos reflete claramente o baixo nível, econômico e educacional das populações e consequente falta de cuidados de higiene por parte dos adultos. Todos os aspectos conduzem à necessidade de maiores investimentos públicos a fim de proporcionar melhorias nas 
condições sociais, econômicas, culturais e de infraestrutura sanitária, minimizando a possibilidade de contaminação, transmissão e efeitos patogênicos das enteroparasitoses.

\section{REFERÊNCIAS}

CARVALHO-COSTA, F. A.; GONÇALVES, A. Q.; LASSANCE, S. L.; NETO, L. M. S.; SALMAZO, C. A. A.; BÓIA, M. N.. Giardia lamblia and other intestinal parasitic infections and their relationships with nutritional status in children in Brazilian Amazon. Revista do Instituto de Medicina Tropical de Sao Paulo, v.49, n.3, p.3-19, 2007. DOI: http://doi.org/10.1590/S0036-46652007000300003

DUNCAN, B. B.; SHMIDT, M. I.; GIUGLIANI, E. R. J.; DUCAN, M. S.; GIUGLIANI, C.. Medicina Ambulatorial: Condutas de Atenção Primária Baseadas em Evidencias. 4 ed. Porto Alegre: Artmed, 2013.

FERREIRA, G. R.; ANDRADE, C. F. S.. Alguns aspectos socioeconômicos relacionados a parasitoses intestinais e avaliação de uma intervenção educativa em escolares de Estiva Gerbi, SP. Revista da Sociedade Brasileira de Medicina Tropical, v.38, n.5, p.402-405, 2005. DOI: http://doi.org/10.1590/S0037-86822005000500008

FERREIRA, M. U.; FERREIRA, C. S.; MONTEIRO, C. A.. Tendência secular das parasitoses intestinais na infância na cidade de São Paulo (1984-1996). Revista de Saude Publica, v.34, n.6 SUPPL., p.73-82, 2000. DOI: http://doi.org/10.1590/S0034-89102000000700010

FONSECA, E. O. L.; TEIXEIRA, M. G.; BARRETO, M. L.; CARMO, E. H.; COSTA, M. C. N.. Prevalência e fatores associados às geo-helmintíases em crianças residentes em municípios com baixo IDH no Norte e Nordeste brasileiros. Cadernos de Saúde Pública, v.26, n.1, p.143-152, 2010. DOI: http://doi.org/10.1590/S0102-311X2010000100015

HOFFMAN, W. A.; PONS, J. A.; JANER, J. L.. The sedimentation-concentration method in schistosomiasis mansoni. Puerto Rico J Public Health, v.9, p.281-298, 1934.

KULIK, R. A.; FALAVIGNA, D. L. M.; NISHI, L.; ARAUJO, S. M.. Blastocystis $\mathrm{sp}$. and other intestinal parasites in hemodialysis patients. Brazilian Journal of Infectious Diseases, v.12, n.4, p.338-341, 2008. DOI: http://doi.org/10.1590/S1413-86702008000400017

LACERDA, M.. g100: Municípios populosos com baixa receita per capita e alta vulnerabilidade Socioeconômica. Brasília: Frente Nacional de Prefeitos, 2017.

LANDER, R. L.; LANDER, A. G.; HOUGHTON, L.; SHEILA, M. W.; COSTA-RIGEIRO, H.; BARRETO, D. L.; MATTOS, A. P.; GIBSON, R. S.. Factors influencing growth and intestinal parasitic infections in preschoolers attending philanthropic daycare centers in Salvador, Northeast Region of Brazil. Cadernos de Saúde Pública, v.28, n.11, p.2177-2188, 2012. DOI: http://doi.org/10.1590/S0102-311X2012001100017

LARRÉ, A. B.; BÜRGIE, C. D.; ENGROFF, P.; CARLI, G. A.. Prevalência de Parasitoses em Idosos residentes e trabalhadores de Instituições de Longa Permanência na Região Metropolitana de Porto Alegre e na Região Serrana do Rio Grande do Sul. O Mundo da Saúde, v.39, n.1, p.84-
91, 2014. DOI: http://doi.org/10.15343/01047809.201539018491

LIANG, C.; CHAN, L.; CHOU, T.; CHIANG, F.; YEN, C.; CHEN, P.; DING, H.; LIN, R.. Brazilein from Caesalpinia sappan L . Antioxidant Inhibits Adipocyte Differentiation and Induces Apoptosis through Caspase-3 Activity and Anthelmintic Activities against Hymenolepis nana and Anisakis simplex. Evidence-Based Complementary and Alternative Medicine, v.2013, p.1-14, 2013. DOI: http://doi.org/10.1155/2013/864892

LUDWIG, K. M.; FREI, F.; FILHO, F. A.; RIBEIRO-PAES, J. T.. Correlação entre condições de saneamento básico e parasitoses intestinais na população de Assis, Estado de São Paulo. Revista da Sociedade Brasileira de Medicina Tropical, v.32, n.5, p.3-12, 1999. DOI: http://doi.org/10.1590/S0037$\underline{86821999000500013}$

MACHADO, E. R.; SOUZA, T. S.; COSTA, J. M.; COSTA-CRUZ, J. M.. Enteroparasites and commensals among individuals living in rural and urban areas in Abadia dos Dourados, Minas Gerais state, Brazil. Parasitologia Latinoamericana, v.63, n.1, p.34-39, 2008. DOI:

http://doi.org/10.4067/S0717-77122008000100006

MONTEIRO, C. A.; CHIEFFI, P. P.; BENICIO, M. H. D.; DIAS, R. M. S.; TORRES, D. M. A. G. V.; MANGINI, A. C. S.. Estudo das condições de saúde das crianças do Município de São Paulo ( Brasil ), 1984 / 1985 . VII - Parasitoses Intestinais. Revista de Saúde Pública, v.22, n.1, p.8-15, 1988. DOI: http://doi.org/10.1590/S0034-89101988000100002

PEREIRA, M. G. C.; ATWILLI, E. R.; BARBOSA, A. P.. Prevalence and associated risk factors for Giardia lamblia infection among children hospitalized for diarrhea in Goiânia, Goiás State, Brazil. Resvista do Instituto de Medicina Tropical de São Paulo, v.49, n.3, p.139-145, 2007. DOI: http://doi.org/10.1590/S0036-46652007000300002

PRESTES, L. F.; JESKE, S.; VELLEDA, C.. Contaminação do solo por geohelmintos em áreas públicas de recreação em municípios do Sul do Rio Grande do Sul (RS), Brasil. Revista de Patologia Tropical, v.44, n.2, p.155-162, 2015. DOI: http://doi.org/10.5216/rpt.v44i2.36645

RIVERO, Z.; BRACHO, Á.; CALCHI, M.; DÍAZ, I.; ACURERO, E.; MALDONADO, A.; GLENIS, C.; ARRÁIZ, N.; CORZO, G.. Detección y diferenciación de Entamoeba histolytica y Entamoeba dispar mediante reacción en cadena de la polimerasa en individuos de una comunidad del Estado Zulia, Venezuela. Cadernos de Saúde Pública, v.25, n.151-159, p.151-159, 2009. DOI: http://doi.org/10.1590/S0102$\underline{311 \times 2009000100016}$

ROSALES, T. F. L.; MALHEIROS, A. F.. Contaminação Ambiental por enteroparasitas presentes em fezes de cães em uma região do Pantanal. O Mundo da Saúde, v.4, n.3, p.368-377, 2017. DOI: http://doi.org/10.15343/0104$\underline{7809.20174103368377}$ 
SANTOS, S. A.; MERLINI, L. S.. Prevalência de enteroparasitoses na população do município de Maria Helena , Paraná. Ciência \& Saúde Coletiva, v.15, n.3, p.899905, 2010. DOI: http://doi.org/10.1590/S1413$\underline{81232010000300033}$

SANTOS, V. M.; SILVA, M. O.; BERNARDES, J. M. G.; LIMA, M. A.. Nódulo granulomatoso com Enterobius vermicularis em epíploon simulando metástase de câncer de ovário. Revista da Sociedade Brasileira de Medicina Tropical, v.35, n.2, p.191-193, 2002. DOI: http://doi.org/10.1590/S0037$\underline{86822002000200010}$

SILVA, J. C.; FURTADO, L. F. V.; FERRO, T. C.; BEZERRA, K. C.; BORGES, E. P.; MELO, A. C. F. L.. Parasitismo por Ascaris lumbricoides e seus aspectos epidemiológicos em crianças do Estado do Maranhão. Revista da Sociedade Brasileira de Medicina Tropical, v.44, n.1, p.100-102, 2011. DOI: http://doi.org/10.1590/S0037-86822011000100022

SMITH, H. M.; DEKAMINSKY, R. G.; NIWAS, S.; SOTO, R. J.; JOLLY, P. E.. Prevalence and Intensity of Infections of Ascaris lumbricoides and Trichuris trichiura and Associated Sociodemographic Variables in Four Rural Honduran Communities. Memorias do Instituto Oswaldo Cruz, v.96, n.3, p.303-314, 2001. DOI: http://doi.org/10.1590/S0074$\underline{02762001000300004}$

SUDRÉ, A. P.; MACEDO, H. W.; SARAMAGO, R. H..

Diagnóstico da estrongiloidíase humana : importância e técnicas. Revista de Patologia Tropical, v.35, n.3, p.173-184, 2006. DOI: http://doi.org/10.5216/rpt.v35i3.1876

TIAGO, P. V.; COSTA, M. S.; PERASSOLO, V.; SOUZA, E. M.; GOMES, M.. Prevalência de parasitores intestinais em pacientes da Unidade Mista de Saúde em Tangará da Serra, Brasil. Revista de Ciências Agro-Ambientais, v.3, p.117-124, 2005.

VISSER, S.; GIATTI, L. L.; CARVALHO, R. A. C.; GUERREIRO, J. C. H.. Estudo da associação entre fatores socioambientais e prevalência de parasitose intestinal em área periférica da cidade de Manaus (AM, Brasil). Ciência \& Saúde Coletiva, v.16, n.8, p.3481-3492, 2011. DOI: http://doi.org/10.1590/S1413-81232011000900016

A CBPC - Companhia Brasileira de Produção Científica (CNPJ: 11.221.422/0001-03) detém os direitos materiais desta publicação. Os direitos referem-se à publicação do trabalho em qualquer parte do mundo, incluindo os direitos às renovações, expansões e disseminações da contribuição, bem como outros direitos subsidiários. Todos os trabalhos publicados eletronicamente poderão posteriormente ser publicados em coletâneas impressas sob coordenação da Sustenere Publishing, da Companhia Brasileira de Produção Científica e seus parceiros autorizados. Os (as) autores (as) preservam os direitos autorais, mas não têm permissão para a publicação da contribuição em outro meio, impresso ou digital, em português ou em tradução. 